\title{
Perfil de resistência a antimicrobianos de Staphylococcus spp. isolados de frangos de corte e poedeiras comerciais no Estado de Pernambuco ${ }^{1}$
}

\begin{abstract}
Mércia R. Barros ${ }^{2}$, Mateus M. da Costa ${ }^{3}$, Chirles A. de França ${ }^{3}$, Tomoe N. Saukas², Leonildo B.G. da Silva², Vanessa Anny S. Silva², Raíssa V. Cavalcante ${ }^{2}$ e Rinaldo A. Mota ${ }^{2}$

ABSTRACT.- Barros M.R., Costa M.M., França C.A., Saukas T.N., Silva L.B.G., Silva V.A.S., Cavalcante R.V. \& Mota R.A. 2011. [Resistance of Staphylococcus spp., to antimicrobials isolated from broilers and commercial layers in the State of Pernambuco, Brazil.] Perfil de resistência a antimicrobianos de Staphylococcus spp., isolados de frangos de corte e poedeiras comerciais no Estado de Pernambuco. Pesquisa Veterinária Brasileira 31(8):672-676. Departamento de Medicina Veterinária, Universidade Federal Rural de Pernambuco, Av. Dom Manuel de Medeiros s/n, Recife, PE 52171900, Brazil. E-mail: rinaldo.mota@hotmail.com

This study had the objective of researching Staphylococcus spp. on healthy broilers and commercial broilers and layers, with clinical respiratory signs. Swabs were taken from the infraorbital sinus of 55 healthy broilers, 35 with respiratory signs and 30 commercial layers also with respiratory signs. Each sample was composed of a pool of five birds, totaling 24 collected samples from 24 commercial flocks. The bacteriological exam was used for the isolation, with a later evaluation of the morphological, tintorial and biochemical characteristics to determine the species. The production of hemolysis, formation of a biofilm in Congo Red Agar, detection of the gene mecA by the PCR and susceptibility to 13 antimicrobial drugs, was verified. From the 24 processed samples, 16 Staphylococcus spp. were obtained isolates, five samples were coagulasis-positive (SCP) and 11 coagulasis-negative (SCN). As to hemolysis and the formation of biofilm tests three samples presented themselves to be hemolytic and six were positive, respectively. In the PCR evaluation for the detection of the mecA gene, all isolates showed negative results. It was observed that $15 \mathrm{E}$ coli isolates were resistant to five or more antibiotics, and that the associated drugs presented better sensibility. The resistance to antimicrobials and biofilm productive strains can interfere in the therapeutic response of birds that present clinical signs.
\end{abstract}

INDEX TERMS: Staphylococus spp., birds, genes, antimicrobial, resistance.

RESUMO.- Este estudo foi realizado com o objetivo de pesquisar Staphylococcus spp. de frangos de corte sadios e frangos de corte e poedeiras comerciais que apresentassem sinais clínicos respiratórios. Foram colhidos swabs dos seios infra-orbitários de 55 frangos de corte sadios, 35 com sinais respiratórios, e 30 poedeiras comerciais também com sinais

\footnotetext{
${ }^{1}$ Recebido em 21 de março de 2011.

Aceito para publicação em 10 de abril de 2011.

Trabalho extraído da Tese de Doutorado do Programa de Pós-Graduação em Ciência Veterinária, Universidade Federal Rural de Pernambuco (UFRPE), Recife/PE, Brasil.

${ }^{2}$ Departamento de Medicina Veterinária, UFRPE, Av. Dom Manuel de Medeiros s/n, Dois Irmãos, Recife, PE 52171900. *Autor para correspondência: rinaldo.mota@hotmail.com

${ }^{3}$ Laboratório de Microbiologia e Imunologia Animal, Campus de Ciências Agrárias, Universidade Federal do Vale do São Francisco, Rua José de Sá Manissoba s/n, Centro, Petrolina, PE 56304-970, Brasil.
}

respiratórios. Cada amostra foi composta por um "pool" de cinco aves, totalizando 24 amostras coletadas de 24 granjas comerciais. Para o isolamento foi utilizado o exame bacteriológico, com posterior avaliação das características morfológicas, tintoriais e bioquímica para determinação da espécie. Verificou-se a produção de hemólise, formação de biofilme em ágar Vermelho Congo (ACR), detecção do gene mecA pela PCR e avaliação da suscetibilidade a 13 drogas antimicrobianas. Das 24 amostras processadas, foram isolados 16 Staphylococcus, cinco isolados foram coagulase-positiva (SCP) e 11 coagulase-negativa (SCN), e nos testes de hemólise e formação de biofilme, três isolados apresentaram-se hemolíticos e seis foram positivos, respectivamente. $\mathrm{Na}$ avaliação por meio da PCR, para detecção do gene mecA todos os isolados apresentaram resultados negativo. Observaram-se que 15 isolados foram resistentes a cinco ou mais antibióticos, e que as drogas associadas apresentaram melhor perfil de sensibi- 
lidade. A resistência a antimicrobianos e cepas produtoras de biofilme podem interferir na resposta terapêutica de aves que apresentam sinais clínicos.

TERMOS DE INDEXAÇÃO: Staphylococus spp., aves, genes, resistência antimicrobiana.

\section{INTRODUÇÃO}

A Estafilococose é uma doença frequente nas aves. A maior parte das infecções é causada por Staphylococcus coagulase positiva, especialmente Staphylococcus aureus, contudo outros Staphylococcus coagulase negativos também estão envolvidos em infecções (Scalan \& Hargis 1989, Jordan 1996, Awan \& Matsumoto 1998, McNamee et al. 1998).

A patogênese da doença é atribuída à combinação de diversos fatores celulares e extracelulares, sendo a formação de biofilme um dos principais mecanismos para a infecção bacteriana persistente ou crônica (Costerton et al. 1999). Arciola et al. (2002) relataram que a produção de biofilme está associada à reduzida sensibilidade aos antimicrobianos.

O uso de antimicrobianos na alimentação animal pode contribuir com a resistência de Staphylococcus (Aarestrup et al. 2000), e para a seleção de cepas resistentes às drogas (Awan \& Matsumoto, 1998). Os agentes antimicrobianos são amplamente utilizados no tratamento e controle das infecções estafilocócicas. Entretanto, poucos estudos têm determinado a ocorrência da resistência antimicrobiana e a presença de genes de resistência de isolados de aves (Aarestrup et al. 2000).

De acordo com Lee (2003) a resistência dos Staphylococcus spp. à oxacilina está relacionada com a presença do gene mecA, que é considerado um determinante genético, que torna os microorganismos intrinsecamente resistentes também a outras drogas. Os genes icaA e icaD presentes em $S$. aureus e $S$. epidermidis estão associados à formação de biofilme (Arciola et al. 2001, 2002), que é um importante fator de virulência e está associado à aderência; podendo reduzir a resposta imune, interferindo com os mecanismos de defesa do hospedeiro (Bernardi et al. 2007).

Objetivou-se com este estudo pesquisar o perfil de resistência a antimicrobianos e a ocorrência de genes de resistência, além da produção de biofilme em isolados de Staphylococcus spp. provenientes de frangos de corte e poedeiras comerciais no Estado de Pernambuco.

\section{MATERIAL E MÉTODOS}

O protocolo experimental deste estudo seguiu os Princípios Éticos na Experimentação Animal, adotados pelo Colégio Brasileiro de Experimentação Animal e foi aprovado pela Comissão de Ética no Uso de Animais da Universidade Federal Rural de Pernambuco (CEUAUFRPE), Processo 23082.001526.

Amostragem. Foram utilizadas 11 granjas de frangos de corte com aves sadias e sete com sinais clínicos, além de seis granjas de poedeiras comerciais com sinais clínicos de doença respiratória. 0 número de propriedades estudadas correspondeu a aproximadamente $20 \%$ do total de granjas no Estado de Pernambuco. 0 material biológico foi colhido dos seios infra-orbitários de 55 frangos de corte sadios, 35 frangos de corte com sinais clínicos e de 30 poedeiras comerciais com sinais clínicos respiratórios. Cada amostra foi constituída por um "pool" de cinco aves, totalizando 24 amostras.
Exame bacteriológico. Os swabs obtidos dos seios infraorbitários foram semeados em ágar base acrescido de sangue desfribinado de ovino a $5 \%$ e as placas foram incubadas a $37^{\circ} \mathrm{C} \mathrm{em}$ aerobiose por até 48 horas. Em seguida realizou-se a coloração do Gram e o teste de catalase para diferenciação de Staphylococcus e Streptococcus. Posteriormente foram realizadas as provas de coagulase para diferenciação entre Staphylococcus coagulase positiva e negativa. Para a identificação das espécies foram realizadas provas bioquímicas como urease, esculina, ágar púrpura base (PAB), glicose semi-sólida (GSS), manitol semi-sólido (MSS) e ágar DNAse (Quinn et al. 1994).

Produção de biofilme. Para estudar a capacidade em produzir biofilme todos os isolados foram semeados em Ágar Vermelho Congo (ACR) e posteriormente incubados a $37^{\circ} \mathrm{C}$ por até $48 \mathrm{~h}$. Após o isolamento, as colônias foram avaliadas quanto à coloração, sendo consideradas positivas aquelas que apresentaram coloração negra (Freeman et al. 1989). Como controle positivo da reação foi utilizado uma cepa de Staphylococcus aureus da Americam Type Culture Collection (ATCC 25923).

Extração de DNA. Todos os isolados foram submetidos à extração de DNA utilizando-se a metodologia descrita por Costa et al. (2010).

Reações e ciclos da PCR. Para reação de amplificação do DNA foram utilizados iniciadores específicos para detecção do gene mecA (Murakami et al. 1991). A reação de PCR constou de $15 \mu \mathrm{L}$ de água ultrapura (Milli-Q), 2,5 $\mu \mathrm{L}$ de Tampão PCR $1 \mathrm{X}, 1 \mu \mathrm{L}$ de $\operatorname{MgCl}_{2}(2 \mu \mathrm{M})$, $1 \mu \mathrm{L}$ de dNTP mix $(0,4 \mu \mathrm{M}$ de cada), $1 \mu \mathrm{L}$ de cada "primer" $(0,4 \mu \mathrm{M})$, $0,5 \mu \mathrm{L}$ de Taq DNA Polimerase $(2,5 \mathrm{U} / \mu \mathrm{L})$ e $3 \mu \mathrm{L}$ do DNA extraído, obtendo-se um volume final de $25 \mu \mathrm{L}$. A reação foi pré-aquecida a $94^{\circ} \mathrm{C}$ por 1 min. seguida de 15 ciclos de desnaturação a $94^{\circ} \mathrm{C}$ por 30 seg., anelamento a $68^{\circ} \mathrm{C}$ por $30 \mathrm{seg}$ e extensão a $72^{\circ} \mathrm{C}$ por $30 \mathrm{seg}$. Foi acrescida uma segunda etapa com 20 ciclos de $94^{\circ} \mathrm{C}$ por $30 \mathrm{seg}$. $60^{\circ} \mathrm{C}$ por $30 \mathrm{seg} .72^{\circ} \mathrm{C}$ por $30 \mathrm{seg}$. e acrescentada uma extensão final a $72^{\circ} \mathrm{C}$ por $2 \mathrm{~min}$ (Kearns et al. 1999). As amostras foram analisadas em gel de agarose a 1,3\% e corado com brometo de etídio (5\%) e os fragmentos obtidos foram visualizados sob luz ultravioleta. Utilizou-se o marcador de peso molecular 100pb Ladder ${ }^{\circledR}$ (Ludwig Biotec, Rio Grande do Sul).

Teste de resistência a antimicrobianos. Todos os isolados de Staphylococcus spp. foram submetidos ao antibiograma utilizando-se a técnica de difusão em discos (Bauer et al. 1966) frente aos antimicrobianos: Amoxicilina $(10 \mu \mathrm{g})$, cefalexina $(30 \mu \mathrm{g})$, clortetraciclina $(15 \mu \mathrm{g})$, enrofloxacina $(5 \mu \mathrm{g})$, lincomicina $(2 \mu \mathrm{g})$, norfloxacina $(10 \mu \mathrm{g})$, oxitetraciclina $(5 \mu \mathrm{g})$, tiafenicol $(30 \mu \mathrm{g})$, trimetoprimsulfadiazina $(30 \mu \mathrm{g})$, trimetoprimsulfadiazinaclortetraciclina $(30 \mu \mathrm{g})$, trimetoprimsulfametoxazolclortetraciclina $(30 \mu \mathrm{g})$, trimetoprimsulfadiazinadoxicilina $(30 \mu \mathrm{g})$ e trimetoprimsulfametoxipiridazinaeritromicina $(10 \mu \mathrm{g})$. A interpretação dos resultados foi realizada de acordo com as recomendações do fabricante.

Análise estatística. Para esse estudo realizou-se análise estatística descritiva calculando-se a frequência absoluta e relativa (Sampaio 1998).

\section{RESULTADOS}

Do total de amostras estudadas observou-se isolamento de Staphylococcus em 66,67\% (16/24), sendo cinco $(31,25 \%)$ identificados como Staphylococcus hyicus, três $(18,75 \%)$ S. lentus, três (18,75\%) S. gallinarum, um (6,25\%) S. chromogenes, um $(6,25 \%)$ S. intermedius, um $(6,25 \%)$ S. saprophyticus e dois $(12,5 \%)$ S. epidermidis. Desses isolados, seis foram obtidos de frangos de corte sem sinais clínicos, oito de frangos de corte com sinais clínicos respiratórios e dois de poedeiras comerciais com sinais clínicos respiratórios (Quadro 1). 
Quadro 1. Staphylococcus spp., isolados dos seios infraorbitários de frangos de corte e de poedeiras comerciais no Estado de Pernambuco

\begin{tabular}{cccccccc}
\hline SC & hyicus & lentus & $\begin{array}{c}\text { galli- } \\
\text { narum }\end{array}$ & $\begin{array}{c}\text { chromo- } \\
\text { genes }\end{array}$ & $\begin{array}{c}\text { inter- } \\
\text { medius }\end{array}$ & $\begin{array}{c}\text { sapho- } \\
\text { phyticus }\end{array}$ & $\begin{array}{c}\text { epider- } \\
\text { midis }\end{array}$ \\
\hline FC SSC & 3 & 1 & 0 & 1 & 0 & 0 & 1 \\
FC CSC & 2 & 2 & 2 & 0 & 1 & 1 & 0 \\
Pc CSC & 0 & 0 & 1 & 0 & 0 & 0 & 1
\end{tabular}

Sistema de Criação; Fc SSC = Frangos de corte sem sinais clínicos; Fc CSC = Frangos de corte com sinais clínicos; Pc CSC = Poedeiras comerciais com sinais clínicos.

Quadro 2. Perfil de resistência dos isolados de Staphylococcus spp., isolados dos seios infra-orbitários de frangos de corte e de poedeiras comerciais no Estado de Pernambuco

\begin{tabular}{lcc}
\hline \multicolumn{1}{c}{ Antibióticos } & \multicolumn{2}{c}{ Interpretação } \\
\cline { 2 - 3 } & F.A. & F.R. (\%) \\
\hline Amoxicilina $(10 \mu \mathrm{g})$ & 14 & 87,5 \\
Cefalexina $(30 \mu \mathrm{g})$ & 9 & 56,3 \\
Clortetraciclina $(15 \mu \mathrm{g})$ & 15 & 93,8 \\
Enrofloxacina $(5 \mu \mathrm{g})$ & 11 & 68,8 \\
Lincomicina $(2 \mu \mathrm{g})$ & 15 & 93,8 \\
Norfloxacina $(10 \mu \mathrm{g})$ & 10 & 62,5 \\
Oxitetraciclina $(5 \mu \mathrm{g})$ & 15 & 93,8 \\
Tiafenicol $(30 \mu \mathrm{g})$ & 10 & 62,5 \\
Trimetoprimsulfadiazina $(30 \mu \mathrm{g})$ & 8 & 50,0 \\
Trimetoprimsulfadiazinaclortetraciclina $(30 \mu \mathrm{g})$ & 10 & 62,5 \\
Trimetoprimsulfadiazinadoxicilina $(30 \mu \mathrm{g})$ & 8 & 50,0 \\
Trimetoprimsulfametoxazolclortetraciclina $(30 \mu \mathrm{g})$ & 7 & 43,8 \\
Trimetoprimsulfametoxipiridazinaeritromicina $(10 \mu \mathrm{g})$ & 7 & 43,8
\end{tabular}

F.A. = Frequência absoluta; F.R. = Frequência relativa.

Quanto à capacidade de coagular o plasma de coelho, 11 $(68,75 \%)$ isolados foram coagulase-negativa (SCN) e seis $(37,5 \%)$ coagulase-positiva (SCP). Dois isolados SCN (S. epidermidis) e um SCP (S. intermedius) apresentaram-se positivos no teste de hemólise.

No teste de produção de biofilme, seis $(37,5 \%)$ isolados foram positivos, sendo dois Staphylococcus hyicus, um $S$. lentus, dois S. gallinarum, um S. saprophyticus e 10 (62,5\%) foram negativos. Observou-se também que os isolados produtores de biofilme apresentaram multirresistência a diferentes grupos de antimicrobianos.

0 perfil de resistência aos antimicrobianos demonstraram que $15(93,75 \%)$ isolados foram resistentes a cinco ou mais antibióticos, apresentando também alta resistência a antibióticos como clortetraciclina, oxitetraciclina e lincomicina $(93,8 \%)$, amoxicilina $(87,5 \%)$, enrofloxacina $(68,8 \%)$, norfloxacina e tiafenicol (62,5\%), enquanto que a utilização de antibióticos utilizados em associação como trimetoprim ou sulfas apresentou perfil de sensibilidade variando de $43,8 \%$ a 50,0\% exceto para Trimetoprimsulfadiazinaclortetraciclina que apresentou 62,5\% de resistência (Quadro 2).

Os isolados de frangos de corte apresentaram resistência à clortetraciclina, lincomicina e oxitetraciclina $(93,8 \%)$, amoxicilina $(87,5 \%)$, enrofloxacina $(68,8 \%)$, norfloxacina, tiafenicol e trimetoprimsulfadiazinaclortetraciclina $(62,5 \%)$, cefalexina $(56,3 \%)$, trimetoprimsulfadiazina e trimetoprimsulfadiazinadoxicilina $(50,0 \%)$ e trimetoprimsulfametoxazolclortetraciclina e trimetoprimsulfametoxipiridazinaeritromicina $(43,8 \%)$. Observou-se ainda que dois isolados de poedeiras comerciais apresentaram resistência à maioria dos antimicrobianos de diferentes grupos. No entanto, no teste para detecção do gene mecA relacionado à resistência à meticilina $\mathrm{e}$ oxacilina todos os isolados foram negativos.

\section{DISCUSSÃO}

Algumas espécies de Staphylococcus são habitantes normais da pele e mucosas dos animais. Nas aves, S. aureus é conhecido por causar várias doenças como septicemia aguda, osteomielite crônica (Skeeles 1997), além de salpingite, ooforite, onfalite, artrite, conjuntivite, blefarite, foliculite, bursite, dermatite gangrenosa e celulite (Ferreira \& Ferreira 2000). Estudo realizado por Takeuchi et al. (1985) demonstrou que $S$. hycus esteve presente na pele e cavidade nasal de 55 (19\%) galinhas saudáveis criadas em granjas no Japão, sendo 17,9\% na pele e 6,2\% na cavidade nasal. Awan \& Matsumoto (1998) também isolaram várias espécies de Staphylococcus de frangos de corte e detectaram $S$. aureus e $S$. intermedius em articulação das aves, apresentando artrite. No Brasil, mais especificamente na região Nordeste, não foram encontrados trabalhos publicados sobre o envolvimento e os prejuízos econômicos causados pela infecção por Staphylococcus spp. em frangos de corte e poedeiras comerciais. Dessa forma este estudo reveste-se de importância epidemiológica, pois determinou as espécies de Staphylococcus presentes nas vias aéreas respiratórias superiores de frangos de corte e poedeiras comerciais. Além disso, observou-se que a maioria dos isolados envolvidos na doença respiratória são SCN, confirmando a participação dessas variantes na doença aviária. Apesar da maior parte das infecções causadas por Staphylococcus em animais ser por SCP, especialmente $S$. aureus, são crescentes os relatos da participação dos SCN em infecções em frangos de corte (Scalan \& Hargis 1989, Awan \& Matsumoto 1998, McNamee et al. 1998).

De acordo com alguns autores, apesar de Staphylococcus spp. serem considerados oportunistas ou secundários (Jonsson \& Wadstrom 1993), a doença ocorre na maioria das vezes quando há queda na resistência devido à infecção por outros patógenos, imunossupressão e lesões na pele ou nas mucosas. Dependendo da capacidade de expressão de fatores de virulência pode ocorrer uma infecção localizada ou septicemia, devido à alta capacidade do microorganismo migrar da pele ou mucosa para os tecidos internos (Ferreira \& Ferreira 2009). Por esse motivo a identificação dos fatores de virulência dessa bactéria é importante para decidir sobre o tratamento que deverá ser instituído no lote (Arciola et al. 2001). A despeito desse aspecto, observou-se ainda no presente estudo a produção de biofilme por alguns isolados obtidos dos seios infra-orbitários de frangos de corte e poedeiras comerciais. Sabe-se que algumas espécies de estafilococos produzem um muco ou biofilme que permite à bactéria aderir às superfícies, sendo importante para a sua colonização. A produção de biofilme é considerada como um fator de virulência (Veenstra et al. 1996, Vuong \& Otto 2002), tornandoas menos acessíveis ao sistema de defesa do organismo e aos antimicrobianos (Arciola et al. 2002, Hume et al. 2004). Esse mecanismo inibe a quimiotaxia, fagocitose, proliferação de linfócitos e limita a atuação dos macrófagos (Vasudevan et al. 2003). 
Alguns estudos têm sugerido que a produção de cápsula facilita a aderência bacteriana através de seus componentes biopolímeros, contribuindo para a formação de biofilme (Peters \& Pulverer 1984). Sendo assim, os microorganismos que produzem biofilme são mais resistentes à ação de agentes físicos e químicos como os utilizados nos procedimentos de higienização (Rickard et al. 2003, Marques 2005). A formação de biofilme é considerada como um dos principais fatores para infecção bacteriana persistente ou crônica (Costerton et al. 1999). Recentemente foi demonstrado que S. aureus e S. epidermidis contém o operon ica responsável pela produção de biofilme (Arciola et al. 2001). Destaca-se também, no presente estudo, a produção de biofilme por amostras de SCN em aves comerciais no estado de Pernambuco, demonstrando a presença desse fator de virulência geralmente relatado para amostras SCP. Observou-se também que as amostras produtoras de biofilme apresentaram multirresistência a vários grupos de antimicrobianos.

0 coeficiente de resitência a antimicrobianos, inclusive de multirresistência observada para amostras isoladas em frangos de corte e poedeiras comerciais a vários antimicrobianos utilizados na avicultura comercial é preocupante para os plantéis estudados. Esses antimicrobianos são largamente utilizados no tratamento e controle de infecções bacterianas nas granjas. Além disso, são administrados há muitos anos, não só para controlar e prevenir a doença, mas também para promover o crescimento e melhorar a eficiência alimentar das aves (Geonaras \& Holy 2001, Bertolatti et al. 2003). Dessa forma, a resistência de cepas produtoras de biofilme pode interferir na resposta terapêutica de aves que apresentam sinais clínicos de doença respiratória. É importante ressaltar ainda a necessidade de se investigar a presença de genes relacionados com a resistência às drogas utilizadas na avicultura.

Informações sobre a prevalência de Staphylococcus resistentes a antimicrobianos na avicultura industrial nos Estados Unidos é escassa, sendo necessária para avaliar os riscos da utilização de antimicrobianos na criação animal e suas consequências na saúde pública (Simjee et al. 2007). No Brasil, pouco se sabe sobre os impactos do uso de antibióticos na avicultura comercial e os danos gerados à saúde animal e pública. A ocorrência de resistência antimicrobiana entre isolados recentes e antigos indica que a resistência aos antimicrobianos em estafilococos de origem aviária aumentou ao longo do tempo em indústrias avícolas da Bélgica e isto pode ser devido ao uso frequente de agentes antimicrobianos na criação de aves (Geonaras \& Holy 2001, Bertolatti et al. 2003).

Poucos estudos têm determinado a ocorrência da resistência antimicrobiana e a presença de genes de resistência entre isolados de estafilococos em aves de produção comercial (Aarestrup et al. 2000). A disseminação de cepas resistentes de Staphylococcus spp. aviária à eritromicina pode causar um impacto considerável sobre a eficácia do tratamento, controle da doença e a rentabilidade da indústria avícola (Nawaz et al. 1999).

0 fato da eritromicina ser rotineiramente empregada no tratamento de doenças em aves, em longo prazo pode trazer consequências indesejáveis, como desenvolvimento de Staphylococcus spp. resistentes à eritromicina (Mota et al. 2005). De acordo com Sahm (1994) e Lee (2003) a resistência dos
Staphylococcus spp. a oxacilina está relacionada à presença do gene mecA que é considerado um determinante genético, que torna os microorganismos intrinsecamente resistentes também a outras drogas.

Kawano et al. (1996) verificaram que os SCN meticilina resistente isolados de galinhas saudáveis apresentaram resistência para maioria dos antibióticos â-lactâmicos, e alguns isolados foram também resistentes para os macrolídeos e aminoglicosídeos. A maior causa da resistência aos â-lactâmicos é atribuída a presença de uma proteína produzida pelo gene mecA (Warsa et al. 1996, Hartman \& Tomasz, 1984). Apesar da ausência do gene mecA nos isolados de Staphylococcus observou-se neste estudo que os mesmos apresentaram resistência a todos os antimicrobianos. De acordo com Gruthuysen et al. (2005) amostras de Staphylococcus aureus armazenadas sob congelamento demonstraram a perda do gene mecA e que a mesma aumentou de acordo com o tempo de estocagem, ou seja, quanto maior o tempo maior a probabilidade de perda do gene. Demonstrando em seus resultados, que apesar da especificidade da técnica utilizada, a sensibilidade da mesma pode variar conforme a forma de conservação das amostras.

\section{CONCLUSÕES}

Os resultados obtidos neste estudo permitem caracterizar a participação de Staphylococcus spp. na doença respiratória de frangos de corte e poedeiras comerciais no estado de Pernambuco.

O elevado coeficiente de resistência a antimicrobianos observada nos isolados pode comprometer o tratamento da estafilococose, indicando-se a realização de testes de sensibilidade in vitro antes do tratamento nas granjas estudadas.

Agradecimentos.- Ao Conselho Nacional de Desenvolvimento Científico e Tecnológico (CNPq Proc.561360/2008-1) e ao Médico Veterinário Glédiston Posso da empresa ALIVET produtos agropecuários Ltda, pelo apoio financeiro para realização desse estudo.

\section{REFERÊNCIAS}

Aarestrup F.M., Agerso Y., Ahrens P., Jorgensen J.C.O., Madsen M. \& Jensen L.B. 2000. Antimicrobial susceptibility and presence of resistance genes in staphylococci from poultry. Vet. Microbiol. 74:353-364.

Arciola C.R., Collamati S., Donati E. \& Montanaro L. 2001. A rapid PCR methods for detection of slime producing strains of Staphylococcus epidermidis and S. aureus in periprothesis infections. Diagn. Mol. Pathol. 10:130-137.

Arciola C.R., Campoccia D., Gamberini S., Cernellati M., Donati E. \& Montanaro L. 2002. Detection of slime production by means of an optimized congo red agar plate test based on a colorimetric scale in Staphylococcus epidermidis clinical isolates genotyped for ica locus. Biomaterials 23:4233-4239.

Awan M.A. \& Matsumoto M. 1998. Heterogeneity of staphylococci and other bacteria isolated from six-week-old broiler chickens. Poult. Sci. 77:944949.

Bauer A.W., Kirby W.M., Sherris J.C. \& Turck M. 1966. Antibiotics susceptibility testing by a standardized single disk method. Am. J. Clin. Pathol. 45:493-496.

Bernardi A.C.A., Pizzolitto E.L. \& Pizzolitto A.C. 2007. Detecção da produção de slime por estafilococos coagulase-negativa isolados de cateter venoso central. Revta Ciênc. Farm. Básica Apl. 28:57-66.

Bertolatti D., O’Brien F.G. \& Grubb W.B. 2003. Characterization of drug- 
resistant Staphylococcus aureus isolated from poultry processing plants in Western Australia. Int. J. Environ. Hlth Res. 13:43-54.

Costa M.M., Drescher G., Maboni F., Weber S., Schrank A., Vainstein M.H., Schrank I. \& Vargas A.C. 2010. Virulence factors, antimicrobial resistance and plasmid content of clinical and environmental Escherichia coli swine isolates. Arq. Bras. Med. Vet. Zootec. 62:30-36.

Costerton J.W., Stewart P. \& Greenberg P. 1999. Bacterial biofilms: A common cause of persistent infections. Science 284:1318-1322.

Ferreira A.J.P. \& Ferreira C.S.A. 2009. Estafilococose e Estreptococose aviária, p.475-482. In: Berchieri Junior A., Silva E.N., Di Fabio J., Sesti L. \& Zuanaze M.A.F. (Eds), Doenças das Aves. FACTA, Campinas.

Freeman D.J., Falkiner F.R. \& Keane C.T. 1989. New method for detecting slime production by coagulase negative staphylococci. J. Clin. Pathol. 42:872-874.

Geornaras I. \& Holy A.V. 2001. Antimicrobial susceptibilities of isolates of Staphylococcus aureus, Listeria and Salmonella serotypes associated with poultry processing. Int. J. Food. Microbiol. 70:29-35.

Gruthuysen A.V., Loo I.V., Alex V.B., Grauls C.V., Wannet W., Keulen P.V. \& Kluytmans J. 2005. Loss of the mecA gene during storage of methicillinresistant Staphylococcus aureus strains. J. Clin. Microbiol. 43:1361-1365.

Hartman B.J. \& Tomasz A. 1984. Low-affinity penicillin-binding protein associated with b-lactam resistance in Staphylococcus aureus. J. Bacteriol. 158:513-516.

Hume E.B.H., Baveja J., Muir B., Schubert T.L., Kumar N., Kjelleberg S., Griesser H.J., Thissen H., Read R., Poolewarren L.A., Schindhelm K. \& Willcox M.D.P. 2004. The control of Staphylococcus epidermidis biofilm formation and in vivo infection rates by covalently bound furanones. Biomaterials 25:5023-5030.

Jonsson P. \& Wadstrom T. 1993. Staphylococcus, p.21-35. In: Gyles C.L. \& Thoen C.O. (Eds), Pathogens of Bacterial Infections in Animals. $2^{\text {nd }}$ ed. Iowa State University Press, Ames.

Jordan F.T.W. 1996. Staphylococci, p.66-69. In: Jordan F.T.W., Pattison M. (Eds), Poultry Diseases. $4^{\text {th }}$ ed. W.B. Saunders, London,

Kawano J., Shimizu A., Saitoh Y., Yagi M., Saito T. \& Okamoto R. 1996. Isolation of methicillin-resistant coagulase-negative Staphylococci from chickens. J. Clin. Microbiol. 34:2072-2077.

Kearns A.M., Seiders P.R., Wheeler J., Freeman R. \& Steward M. 1999. Rapid detection of methicilin-resistant Staphylococci by multiplex PCR. J. Hosp. Infect. 43:33-37.

Lee J.H. 2003. Methicilin (oxacilin) resistant Staphylococcus aureus strains isolated from major food animals and their potential transmission to humans. Appl. Environ. Microbiol. 69:6489-6494.

Marques C.S. 2005. Formação de biofilmes por Staphylococcus aureus na superfície de aço inoxidável e vidro e sua resistência a sanificantes químicos. Dissertação de Mestrado em Ciência e Tecnologia de Alimentos, Universidade Federal de Lavras, Lavras. 64p.

McNamee P.T., McCullagh J.J., Thorp B.H., Graham D., McCullough S.J.,
McConaghy D. \& Smyth J.A. 1998. Study of leg weakness in two commercial broiler flocks. Vet. Res. 143:131-135.

Mota R.A., Silva K.P.C., Freitas M.F.L., Porto W.J.N. \& Silva L.B.G. 2005. Utilização indiscriminada de antimicrobianos e sua contribuição a multirresis-tência bacteriana. Braz. J. Vet. Res. Anim. Sci. 42:465-470.

Murakami K., Minamide W., Wada K., Nakamura E., Teraoka H. \& Watanabe S. 1991. Identification of methicilin-resistant strains of staphylococci by polimerase chain reaction. J. Clin. Microbiol. 29:2240-2244.

Nawaz M.S., Khan A.A., Khan S.A., Paine D.D., Pothuluri J.V. \& Cerniglia C.E. 1999. Biochemical and molecular characterization of erythromycin resistant avian Staphylococcus spp. isolated from chickens. Poult. Sci. 78:1191-1197.

Peters G. \& Pulverer G. 1984. Pathogenesis and management of Staphylococcus epidermidis 'plastic' foreign body infections. J. Antimicrob. Chemother. 14:67-71.

Quinn P.J., Carter M.E., Markey B.K. \& Carter G.R. 1994. Clinical Veterinary Microbiology. Wolf, London. 648p.

Rickard A.H., Gilbert P., High N.J., Kolenbrander P.E. \& Handley P.S. 2003. Bacterial coaggregation: An integral process in the development of multispecies biofilms. Trends Microbiol. 11:94-100.

Sampaio I.B.M. 1998. Estatística Alicada à Experimentação Animal. UFMG, Belo Horizonte. 221p.

Scanlan C.M. \& Hargis B.M. 1989. A bacteriologic study of scabby-hip lesions from broiler chickens in Texas. J. Vet. Diagn. Invest. 1:170-173.

Sahm D.F. 1994. Streptococci and staphylococci: Laboratory considera-tions for in vitro susceptibility testing. Clin. Microbiol. Newslett. 16:9-13.

Simjee S., McDermont P.F., White D.G., Hofacre C., Berghaus R.D., Carter P.J., Stewart L., Liu T., Maier M. \& Maurer J.J. 2007. Antimicrobial susceptibility and distribution of antimicrobial resistance genes among Enterococcus and coagulase-negative Staphylococcus isolates recovered from poultry litter. Avian Dis. 51:884-892.

Skeeles J.K. 1997. Staphylococcosis, p.247-253. In: Calnek B.W. (Ed.), Diseases of Poultry. Iowa State University Press, Ames.

Takeuchi S., Kobayashi Y., Morozumi T. \& Niibori S. 1985. Isolation and some properties of Staphylococcus hyicus subsp. hyicus from pigs, chickens and cows. Jpn. J. Vet. Sci. 47:841-843.

Veenstra G., Cremers F., Van Dijk H. \& Fleer A. 1996. Ultrastructural organization and regulation of a biomaterial adhesion of Staphylococcus epidermidis. J. Bacteriol. 178:537-41.

Vuong C. \& Otto M. 2002. Staphylococcus epidermidis infections. Microbes Infect. 4:481-489.

Vasudevan P., Nair M.K.M., Annamalai T. \& Venkitanarayana K.S. 2003. Staphylococcus aureus for biofilm formation. Vet. Microbiol 92:179-185.

Warsa U.C., Okubo T. \& Okamoto R. 1996. Antimicrobial susceptibilities and phage typing of Staphylococcus aureus clinical isolates in Indonesia. J. Infect. Chemother. 2:29-33. 